\title{
Sufentanil Subaracnóideo Associado à Bupivacaína Hiperbárica para Analgesia de Parto: É Possível Reduzir a Dose do Opióide? *
}

\section{Spinal Sufentanil Associated to Hyperbaric Bupivacaine: Is it Possible to Decrease Opioid Dose?}

Eduardo Tsuyoshi Yamaguchi, TSA ${ }^{1}$; José Carlos Almeida Carvalho, TSA ${ }^{2}$; Ubirajara Sabbag Fonseca ${ }^{3}$; Jacqueline Toshiko Hirahara ${ }^{4}$; Mônica Maria Siaulys Capel Cardoso, TSA ${ }^{5}$

\section{RESUMO}

Yamaguchi ET, Carvalho JCA, Fonseca US, Hirahara JT, Cardoso MMSC - Sufentanil Subaracnóideo Associado à Bupivacaína Hiperbárica para Analgesia de Parto: É Possível Reduzir a Dose do Opióide?

JUSTIFICATIVA E OBJETIVOS: A adição de bupivacaína isobárica a doses menores de sufentanil por via subaracnóidea promove analgesia de qualidade satisfatória, com menor incidência de efeitos colaterais. O objetivo do estudo foi avaliar a qualidade da analgesia e a incidência de efeitos colaterais de doses reduzidas de sufentanil subaracnóideo associados à bupivacaína hiperbárica em analgesia de parto.

MÉTODO: Foram estudadas prospectivamente 69 gestantes de termo em trabalho de parto. As gestantes foram aleatoriamente divididas em três grupos que receberam, no espaço subaracnóideo, a combinação de $2,5 \mathrm{mg}$ de bupivacaína hiperbárica e $1 \mathrm{ml}$ de solução fisiológica (Grupo Controle); 2,5 mg de bupivacaína hiperbárica e 2,5 $\mu \mathrm{g}$ de sufentanil (Grupo S2,5) ou 2,5 mg de bupivacaína hiperbárica e $5 \mu \mathrm{g}$ de sufentanil (Grupo S5). A dor, de acordo com a escala analógico visual (EAV) de dor e a incidência de efeitos colaterais foram avaliadas a cada 5 minutos nos primeiros quinze minutos e a seguir a cada 15 minutos até o nascimento. O estudo terminava com o nascimento, ou quando a paciente solicitava medicação analgésica de resgate $(E A V>3 \mathrm{~cm})$.

RESULTADOS: Os grupos S2,5 e S5 apresentaram maior duração de analgesia $(67,2 \pm 38,6$ e 78,9 \pm 38,7 minutos, respectivamente) e maior porcentagem de pacientes com analgesia efetiva (100\% e 95,6\%, respectivamente) que o grupo

* Recebido do (Received from) Departamento de Anestesiologia da Faculdade de Medicina da Universidade de São Paulo (FMUSP) e do Hospital e Maternidade Santa Joana

1. Médico Colaborador da Disciplina de Anestesiologia da FMUSP. Anestesiologista do Hospital e Maternidade Santa Joana

2. Diretor Científico do Departamento de Anestesiologia do Hospital e Maternidade Santa Joana

3. $M E_{2}$ da Disciplina de Anestesiologia da FMUSP

4. $M E_{3}$ da Disciplina de Anestesiologia da FMUSP. Anestesiologista do Hospital e Maternidade Santa Joana

5. Doutora em Anestesiologia pela FMUSP. Médica Supervisora da Anestesia Obstétrica do HCFMUSP. Anestesiologista do Hospital e Maternidade Santa Joana

Apresentado (Submitted) em 28 de abril de 2003

Aceito (Accepted) para publicação em 17 de julho de 2003

Endereço para correspondência (Correspondence to)

Dra. Mônica Maria Siaulys Capel Cardoso

Av. Dr. Enéas de Carvalho Aguiar, 255

$8^{\circ}$ Andar PAMB - Divisão de Anestesiologia

05403-900 São Paulo, SP

(C) Sociedade Brasileira de Anestesiologia, 2004
Controle, no qual a duração média de analgesia foi de 35,9 \pm 21,6 minutos $(p<0,05)$ e a porcentagem de pacientes com analgesia efetiva foi de 69,6\% $(p<0,05)$.

CONCLUSÕES: A adição de sufentanil à bupivacaína hiperbárica melhora a qualidade e prolonga a duração da analgesia. Quando associado a 2,5 mg de bupivacaína hiperbárica, não há vantagem de se administrar dose superior a 2,5 $\mu \mathrm{g}$ de sufentanil para alívio da dor do trabalho de parto.

Unitermos: ANALGESIA: parto; ANALGÉSICOS, Opióides: sufentanil; ANESTÉSICOS, Local: bupivacaína; TÉCNICAS ANESTÉSICAS, Regional: combinada, peridural, subaracnóidea

\section{SUMMARY}

Yamaguchi ET, Carvalho JCA, Fonseca US, Hirahara JT, Cardoso MMSC - Spinal Sufentanil Associated to Hyperbaric Bupivacaine: Is it Possible to Decrease Opioid Dose?

BACKGROUND AND OBJECTIVES: The association of isobaric bupivacaine to lower spinal sufentanil dose provides satisfactory analgesia and lower incidence of side effects. This study aimed at evaluating quality of analgesia and incidence of side effects of decreased spinal sufentanil doses associated to hyperbaric bupivacaine for labor analgesia.

METHODS: Participated in this study 69 healthy term pregnant patients in active labor. Patients were randomly allocated in three groups receiving spinal combination of $2.5 \mathrm{mg}$ hyperbaric bupivacaine and $1 \mathrm{~mL}$ saline solution (Control Group); $2.5 \mathrm{mg}$ hyperbaric bupivacaine and $2.5 \mu \mathrm{g}$ sufentanil (S2.5 Group) or $2.5 \mathrm{mg}$ hyperbaric bupivacaine and $5 \mu \mathrm{g}$ sufentanil (S5 Group). Pain, using a visual analogue scale (VAS), and side effects were evaluated at 5-minute intervals for the first 15 minutes and then at 15-minute interval until delivery. Study would end with delivery or when patient requested rescue analgesia (VAS $>3$ $\mathrm{cm}$ ).

RESULTS: Groups S2.5 and S5 presented longer analgesia duration (67.2 \pm 38.6 and $78.9 \pm 38.7$ minutes, respectively) and a larger number of patients with effective analgesia (100\% and $95.6 \%$, respectively) as compared to Control group where mean analgesia duration was $35.9 \pm 21.6$ minutes $(p<0.05)$ with $69.6 \%$ of patients with effective analgesia $(p<0.05)$.

CONCLUSIONS: The association of sufentanil to hyperbaric bupivacaine improves quality and prolongs analgesia duration. When associated to $2.5 \mathrm{mg}$ hyperbaric bupivacaine, there is no benefit in administering more than $2.5 \mu \mathrm{g}$ of sufentanil for labor pain relief.

Key Words: ANALGESIA: labor; ANALGESICS, Opioids: sufentanil; ANESTHETICS, Local: bupivacaine; ANESTHETIC TECHNIQUES, Regional: combined, epidural, spinal block 


\section{INTRODUÇÃO}

O sufentanil é um opióide bastante utilizado em analgesia de parto pelo seu rápido início de ação e alta potência analgésica. Herman e col. ${ }^{1}$ e Arkoosh e col. ${ }^{2}$ demonstraram que a $D E_{50}$ do sufentanil para analgesia de parto como droga única é 2,6 $\mu \mathrm{g}$ (dose necessária para se obter analgesia em $50 \%$ das pacientes) e que a $\mathrm{DE}_{95}$ é $8,9 \mu \mathrm{g}$ (dose necessária para se obter analgesia em $95 \%$ das pacientes), quando administrado no espaço subaracnóideo.

Em analgesia de parto, é freqüente a associação do opióide com o anestésico local. O efeito aditivo e sinérgico dessas drogas permite que se obtenha melhor qualidade de analgesia, com menor incidência de efeitos colaterais ${ }^{3}$.

Estudo dose-resposta da combinação do sufentanil com à bupivacaína isobárica mostra que quando associados à 2,5 mg de bupivacaína, a redução da dose de sufentanil subaracnóideo de $10 \mu \mathrm{g}$ para $2,5 \mu \mathrm{g}$ reduz a intensidade de prurido, sem prejudicar a qualidade da analgesia ${ }^{4}$.

Nossa rotina em analgesia de parto tem sido a associação do sufentanil à bupivacaína hiperbárica no espaço subaracnóideo. A adição do sufentanil hipobárico à bupivacaína hiperbárica resulta em solução hiperbárica.

A baricidade das soluções anestésicas pode modificar de maneira significativa à dispersão dos agentes anestésicos no espaço subaracnóideo, produzindo efeitos clínicos distintos.

O objetivo deste estudo foi analisar a qualidade da analgesia e a incidência de efeitos colaterais proporcionadas pela administração de duas doses diferentes de sufentanil subaracnóideo associados à bupivacaína hiperbárica em analgesia de parto.

\section{MÉTODO}

Após a aprovação das Comissões de Ética em Pesquisa do Hospital das Clínicas da Faculdade de Medicina da Universidade de São Paulo e do Hospital e Maternidade Santa Joana, foi realizado estudo prospectivo, aleatório, duplamente encoberto, em 69 gestantes de termo, hígidas, em trabalho de parto, sem complicações obstétricas ou fetais.

Após monitorização com eletrocardioscópio, aparelho de pressão arterial não invasiva e oximetria de pulso, as pacientes receberam expansão volêmica com $250 \mathrm{ml}$ de solução de Ringer com lactato. A técnica combinada raqui-peridural foi realizada com a paciente na posição sentada, no espaço $L_{2}-L_{3}$ ou $L_{3}-L_{4}$, utilizando-se a técnica de "agulha através de agulha”, sendo que a solução anestésica foi administrada somente após a obtenção de refluxo passivo de líquor através do canhão da agulha. A passagem do cateter no espaço peridural foi realizada imediatamente a seguir. Após o término da punção, as pacientes foram colocadas em decúbito dorsal horizontal com deslocamento uterino para a esquerda. As soluções anestésicas utilizadas foram preparadas por anestesiologista que não estava envolvido com o atendimento da paciente.

No momento em que solicitaram analgesia, as pacientes foram aleatoriamente divididas em 3 grupos, que receberam no espaço subaracnóideo:

- Grupo Controle $(n=23):$ 2,5 mg de bupivacaína a 0,5\% hiperbárica associados a $1 \mathrm{ml}$ de solução fisiológica;

- Grupo S2,5 ( $n=23$ ): 2,5 $\mu$ g de sufentanil associados a 2,5 mg de bupivacaína a $0,5 \%$ hiperbárica e $0,5 \mathrm{ml}$ de solução fisiológica;

- Grupo S5 ( $\mathrm{n}=23$ ): $5 \mu \mathrm{g}$ de sufentanil associados a 2,5 mg de bupivacaína a 0,5\% hiperbárica.

Ador e os possíveis efeitos colaterais (náusea, vômito, prurido) foram avaliados no momento da realização da analgesia, a cada 5 minutos nos 15 primeiros minutos, e a seguir a cada 15 minutos até o nascimento.

A dor foi avaliada utilizando-se escala analógico-visual (EAV) de 0 a $10 \mathrm{~cm}$ (0 - ausência de dore 10 - dor insuportável) por anestesiologista que desconhecia a que grupo pertencia a paciente.

Após os 15 minutos iniciais, em caso de dor maior que $3 \mathrm{~cm}$, administrou-se bolus de $4 \mathrm{ml}$ de concentrações crescentes de bupivacaína a $0,125 \%$ e a $0,25 \%$ a cada 15 minutos pelo cateter peridural até o controle da dor (dor menor que 3 $\mathrm{cm})$.

O estudo encerrava-se no momento da primeira complementação analgésica através do cateter peridural ou no nascimento.

A análise estatística foi realizada com ANOVA (para comparação da idade, peso, altura, dilatação inicial e duração da analgesia entre os grupos), Kruskal-Wallis (EAV inicial e após quinze minutos da injeção subaracnóidea), Qui-quadrado (nascimento antes da necessidade de complementação) e teste Exato de Fisher (náusea, vômito, prurido e complementação nos 15 minutos iniciais), adotando como nível de significância $p<0,05$.

\section{RESULTADOS}

Os grupos foram semelhantes em relação às variáveis antropométricas de idade, peso e altura (Tabela I) e em relação às médias de dilatação cervical inicial (Tabela II).

Tabela I - Variáveis Antropométricas (Média \pm DP)

\begin{tabular}{lccc}
\hline Grupos & $\begin{array}{c}\text { Controle } \\
(\mathrm{n}=23)\end{array}$ & $\begin{array}{c}\mathrm{S} 2,5 \mu \mathrm{g} \\
(\mathrm{n}=23)\end{array}$ & $\begin{array}{c}\mathrm{S5} \mu \mathrm{gg} \\
(\mathrm{n}=23)\end{array}$ \\
\hline Idade (anos) & $26,7 \pm 6,6$ & $28,4 \pm 7,1$ & $24,5 \pm 6,4$ \\
Peso $(\mathrm{kg})$ & $69,9 \pm 11,3$ & $71,5 \pm 10,3$ & $71,7 \pm 16,0$ \\
Altura $(\mathrm{m})$ & $1,60 \pm 0,1$ & $1,59 \pm 0,1$ & $1,60 \pm 0,1$ \\
\hline
\end{tabular}


Tabela II - Dilatação Cervical Inicial, Duração da Analgesia e Eficácia da Analgesia (EAV $<3 \mathrm{~cm}$ ) após 15 minutos

\begin{tabular}{lccc}
\hline Grupos & $\begin{array}{c}\text { Controle } \\
(\mathrm{n}=23)\end{array}$ & $\begin{array}{c}\mathrm{S} 2,5 \mu \mathrm{g} \\
(\mathrm{n}=23)\end{array}$ & $\begin{array}{c}\mathrm{S5} \mu \mathrm{g} \\
(\mathrm{n}=23)\end{array}$ \\
\hline Dilatação inicial (cm) & $5,7 \pm 1,6$ & $5,8 \pm 1,7$ & $5,4 \pm 1,9$ \\
Duração analgesia (min) & $35,9 \pm 21,6^{*}$ & $67,2 \pm 38,6$ & $78,9 \pm 38,7$ \\
EAV <3 cm após 15' (\%) & $69,6^{*}$ & 100 & 95,6 \\
\hline
\end{tabular}

${ }^{*} \mathrm{p}<0,05$, Grupo Controle $<$ Grupo S2,5 = Grupo S5

Os grupos que receberam sufentanil nas doses de $2,5 \mu \mathrm{g}$ ou 5 $\mu \mathrm{g}$ apresentaram maior duração da analgesia e menor necessidade de complementação analgésica após os quinze minutos iniciais que o grupo Controle (Tabela II).

Tabela III - Incidência de Náusea, Vômito e Prurido (Leve ou Intenso)

\begin{tabular}{lccc}
\hline Grupos & $\begin{array}{c}\text { Controle } \\
(\mathrm{n}=23)\end{array}$ & $\begin{array}{c}\mathrm{S} 2,5 \mu \mathrm{g} \\
(\mathrm{n}=23)\end{array}$ & $\begin{array}{c}\mathrm{S} 5 \mu \mathrm{gg} \\
(\mathrm{n}=23)\end{array}$ \\
\hline Náusea (\%) & 4 & 4 & 9 \\
Vômito (\%) & 4 & 4 & 4 \\
Prurido leve (\%) & $0^{*}$ & 34,8 & 47,8 \\
Prurido intenso (\%) & 0 & 0 & 8,7 \\
\hline
\end{tabular}

${ }^{*} \mathrm{p}<0,05$, Grupo S2,5 = Grupo S5 $>$ Grupo Controle

Afigura 1 ilustra a porcentagem de pacientes com EAV menor que $3 \mathrm{~cm}$ em função do tempo. Nos grupos $\mathrm{S} 2,5$ e $\mathrm{S} 5$ houve maior número de nascimentos antes da necessidade de complementação analgésica quando comparados ao Controle (Figura 2).

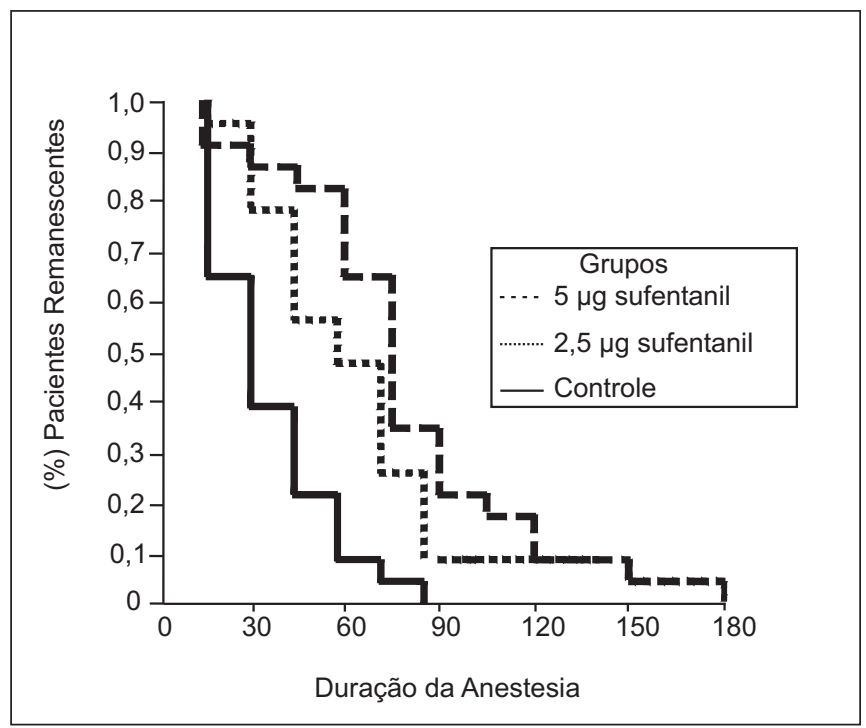

Figura 1 - Porcentagem de Pacientes com Analgesia Efetiva $(E A V<3)$ em Função do Tempo

Revista Brasileira de Anestesiologia

Vol. 54, № 2, Março - Abril, 2004

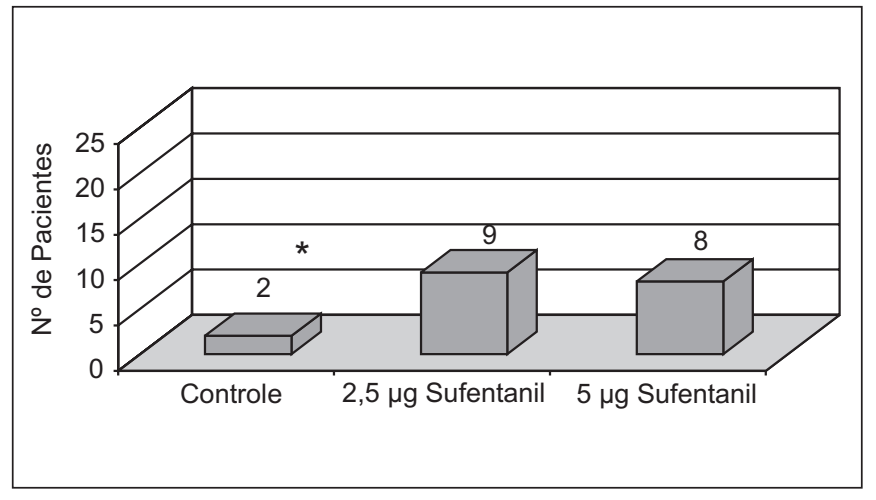

Figura 2 - Número de Nascimentos antes da Necessidade de Complementação Analgésica

${ }^{*} \mathrm{p}<0,05$, Grupo Controle $<$ Grupo S2,5 = Grupo S5

Na instalação da analgesia, as médias de dor de acordo com a EAV eram semelhantes entre os três grupos. No entanto, observou-se que quinze minutos após a injeção inicial da solução anestésica no espaço subaracnóideo as médias de dor no grupo Controle foram significativamente maiores que as encontradas nos grupos que receberam o sufentanil nas doses de 2,5 e $5 \mu \mathrm{g}$ (Figura 3).

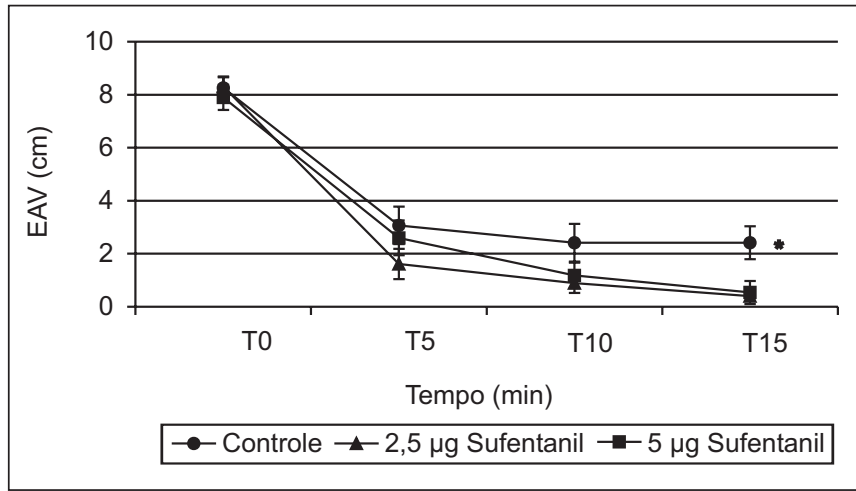

Figura 3 - EAV $(\mathrm{cm})$ Antes e Após os Quinze Minutos Iniciais da Injeção Subaracnóidea

${ }^{*} p<0,05$, Grupo Controle $>$ Grupo S2,5 = Grupo S5

Em relação aos efeitos colaterais, não houve diferença estatisticamente significativa na incidência de náusea e vômitos entre os grupos. A ocorrência de prurido leve foi maior nos grupos S2,5 e S5 que no grupo Controle. O prurido intenso ocorreu em duas pacientes do grupo S5, não havendo diferença estatisticamente significativa dessa variável entre os três grupos (Tabela III).

\section{DISCUSSÃO}

Nossos resultados demonstraram que a adição de sufentanil nas doses de 2,5 $\mu \mathrm{g}$ e $5 \mu \mathrm{g}$ à bupivacaína hiperbárica no espaço subaracnóideo para analgesia de parto determina maior duração e melhor qualidade analgésica que a administração isolada do anestésico local. Estes dados estão de acordo 
com Wong e col. ${ }^{4}$, que em estudo dose-resposta da combinação de opióide e anestésico local, também concluíram que a adição de diferentes doses de sufentanil $(0,25 ; 5 ; 7,5$ e 10 $\mu \mathrm{g})$ à bupivacaína isobárica melhorava a qualidade e prolongava a duração da analgesia de parto.

A grande diferença entre estes dois estudos provavelmente está na baricidade das soluções anestésicas administradas no espaço subaracnóideo. Aadição de 0,5 ou $1 \mathrm{ml}$ de sufentanil (densidade $=0,9933 \mathrm{~g} \cdot \mathrm{ml}^{-1}$ ) a $0,5 \mathrm{ml}$ de bupivacaína hiperbárica a $0,5 \%$ (densidade $=1,026 \mathrm{~g} \cdot \mathrm{ml}^{-1}$ ), a $20^{\circ} \mathrm{C}$ produz soluções hiperbáricas (densidade da solução $=1,002935$ g. $\mathrm{ml}^{-1}$ ). Wong e col. ${ }^{4}$; adicionando diferentes doses de sufentanil à bupivacaína isobárica, trabalharam com soluções hipobáricas.

Sabe-se que modificações nas baricidades das soluções anestésicas administradas no espaço subaracnóideo, especialmente do sufentanil, podem modificar de maneira significativa a dinâmica da dispersão das soluções anestésicas no interior do espaço subaracnóideo e, consequentemente, os seus efeitos clínicos ${ }^{5}$.

Ferouz e col. ${ }^{6}$ demonstraram que a adição de $1 \mathrm{ml}$ de dextrose a $10 \%$ a $1 \mathrm{ml}$ de sufentanil $\left(10 \mu \mathrm{g} \cdot \mathrm{ml}^{-1}\right)$, produzindo, portanto, solução hiperbárica de sufentanil, determinava redução na ocorrência de efeitos colaterais do mesmo (principalmente de prurido intenso) e prejuízo na qualidade da analgesia. Sugeriram, portanto, que os efeitos colaterais dependiam da ação supra-espinhal dos opióides e que a ação analgésica dependia tanto da ação espinhal quanto da ação supra-espinhal.

Apesar de não ter sido comparada diretamente a eficácia analgésica de soluções hipo ou hiperbáricas contendo sufentanil e bupivacaína, provavelmente, neste estudo, eventuais prejuízos na qualidade de analgesia em decorrência da administração de sufentanil hiperbárico, tenham sido corrigidos pela associação com o anestésico local, já que ambas as drogas apresentam efeitos aditivo e sinérgico em relação à analgesia.

É nossa rotina administrar opióide associado ao anestésico local, já que em nosso meio a analgesia de parto freqüentemente é instalada no final do primeiro estágio e início do segundo estágio do trabalho de parto. Estudos anteriores demonstram que a utilização isolada de opióide subaracnóideo não é eficaz para aliviar a dor do trabalho de parto, especialmente à medida que a gestante se aproxima do segundo estágio do trabalho de parto ${ }^{7-10}$.

Entretanto, vale à pena ressaltar que nesse estudo, a indicação da analgesia sempre foi baseada no momento de solicitação pela paciente e não na dilatação cervical. Embora a média da dilatação cervical no momento em que foi solicitada a analgesia estivesse em torno de $5 \mathrm{~cm}$, ocorreu grande variabilidade individual (1 a $9 \mathrm{~cm}$ ), o que reflete a variação individual de tolerância à dor no momento do trabalho de parto.

Optou-se por não realizar a dose-teste com $3 \mathrm{ml}$ de lidocaína a 1,5\% com epinefrina 1:200.000 imediatamente após a injeção da solução anestésica no espaço subaracnóideo e posterior passagem do cateter peridural como proposto por
Wong e col. ${ }^{4}$, já que a dose-teste poderia ter contribuído com a analgesia.

A dose-teste poderia melhorar a qualidade da analgesia de reduzidas doses de sufentanil e bupivacaína subaracnóidea por ação direta da lidocaína no espaço peridural ou de maneira indireta, interferindo na dinâmica da dispersão das soluções anestésicas recentemente injetadas e ainda não fixadas no interior do espaço subaracnóideo (efeitos de massa e de transmissão da pressão peridural para o espaço subaracnóideo).

Cardoso e col. ${ }^{11}$, avaliando as modificações das pressões peridurais decorrentes da injeção do anestésico local em diferentes volumes e velocidades, mostraram que mesmo pequenos volumes como aqueles utilizados na dose-teste, quando injetados rapidamente, determinam picos prescritos no espaço peridural maiores que a administração de volumes maiores em velocidades menores.

Vale ainda ressaltar que a verificação momentânea do correto posicionamento do cateter peridural não garante a permanência do cateter no mesmo espaço por ocasião de manipulações futuras. Vários relatos de migração intravascular e subaracnóidea de cateteres peridurais previamente bem posicionados podem ser encontrados na literatura. Além disso, em analgesia de parto, complicações decorrentes da injeção intravascular de anestésico local por eventual posicionamento do cateter peridural no intravascular raramente ocorrem, pois o anestesiologista está trabalhando com doses e concentrações extremamente reduzidas de anestésico local.

Em relação aos efeitos colaterais, observou-se que a redução da dose de sufentanil de 5 para $2,5 \mu$ g não modificou a ocorrência de náusea, vômito, prurido leve, prurido intenso e nem a duração da analgesia. Esses dados estão de acordo com os encontrados por Wong e col. ${ }^{4}$, que também não encontraram diferenças em relação às incidências de náusea, vômito, prurido e duração da analgesia após a administração de diferentes doses de sufentanil subaracnóideo $(2,5 ; 5 ; 7,5$ e $10 \mu \mathrm{g})$. Em ambos os estudos, as diferenças em relação às incidências de efeitos colaterais e duração da analgesia só foram encontradas entre os grupos que receberam analgesia com a combinação de sufentanil e bupivacaína quando comparados às observadas nos grupos controles, em que a analgesia de parto foi praticada somente com a utilização do anestésico local.

Entretanto, é importante salientar que o tamanho amostral não é suficiente para detectar possíveis diferenças entre as duas doses de sufentanil utilizadas. A análise de poder para as variáveis relacionadas a efeitos colaterais mostra que para se detectar diferença na ocorrência de prurido leve da ordem de $13 \%$ entre os dois grupos, seria necessário analisar grupos de 198 pacientes cada.

Observou-se ainda que o nascimento antes da necessidade de complementação peridural ocorreu em um número maior de pacientes nos grupos que receberam o sufentanil que no controle. Esse fato não necessariamente indica maior velocidade de dilatação cervical com o uso de sufentanil. Provavel- 
mente, está relacionado à maior duração da analgesia nesses grupos em relação à observada no grupo controle. Embora a depressão respiratória já tenha sido relatada com o uso de sufentanil subaracnóideo ${ }^{12-14}$, neste estudo não foi identificado nenhum caso de depressão respiratória após o uso do sufentanil por essa via. Embora, também, o número de pacientes estudadas seja pequeno para se obter qualquer conclusão, provavelmente os fatores que contribuíram para a não ocorrência de tal complicação são: a não utilização de qualquer outra droga depressora do sistema nervoso central por via sistêmica; as soluções anestésicas foram preparadas partindo-se da solução mãe de sufentanil para uso espinhal que contém apenas $5 \mu \mathrm{g}$ da droga por ml e não a partir da solução para uso venoso que contém $50 \mu \mathrm{g}$ da droga por ml (evitam-se portanto eventuais erros de diluição); as doses de sufentanil utilizadas nesse estudo foram menores (2,5 e 5 $\mu \mathrm{g})$ que as utilizadas nos casos em que a depressão respiratória foi encontrada (10 $\mu \mathrm{g})$ e a utilização de soluções hiperbáricas, que provavelmente limita a dispersão cefálica das soluções no espaço subaracnóideo ${ }^{6}$.

Concluindo, a associação de diferentes doses de sufentanil à bupivacaína hiperbárica por via subaracnóidea melhora a qualidade e aumenta a duração da analgesia de parto. Quando associado a 2,5 mg de bupivacaína hiperbárica, não há vantagem em se administrar dose de sufentanil superior a $2,5 \mu \mathrm{g}$.

\section{Spinal Sufentanil Associated to Hyperbaric Bupivacaine: Is it Possible to Decrease Opioid Dose?}

Eduardo Tsuyoshi Yamaguchi, TSA, M.D.; José Carlos Almeida Carvalho, TSA, M.D.; Ubirajara Sabbag Fonseca, M.D.; Jacqueline Toshiko Hirahara, TSA, M.D.; Mônica Maria Siaulys Capel Cardoso, TSA, M.D.

\section{INTRODUCTION}

Sufentanil is a widely used opioid for labor analgesia for its fast onset and high analgesic potency. Herman et al. ${ }^{1}$ and Arkoosh et al. ${ }^{2}$ have shown that sufentanil's $D E_{50}$ for spinal labor analgesia as a single drug is $2.6 \mu \mathrm{g}$ (dose needed for inducing analgesia in $50 \%$ of patients) and that $D E_{95}$ is $8.9 \mu \mathrm{g}$ (dose needed for inducing analgesia in $95 \%$ of patients).

The association of opioids to local analgesics is common in labor analgesia. The additive and synergistic effect of such drugs provides better quality analgesia with lower incidence of side effects ${ }^{3}$.

Adose-response study with the combination of sufentanil and isobaric bupivacaine has shown that, when associated to 2.5 mg bupivacaine, spinal sufentanil dose may be decreased from $10 \mu \mathrm{g}$ to $2.5 \mu \mathrm{g}$ decreasing pruritus intensity without impairing analgesia quality ${ }^{4}$.
Our routine labor analgesia has been the association of spinal sufentanil and hyperbaric bupivacaine. The association of hypobaric sufentanil and hyperbaric bupivacaine results in hyperbaric solution.

Anesthetic solutions baricity may significantly change anesthetic agents spread in the spinal space producing different clinical effects.

This study aimed at evaluating quality of analgesia and incidence of side effects provided by two different spinal sufentanil doses associated to hyperbaric bupivacaine in labor analgesia.

\section{METHODS}

After the Hospital das Clínicas, Faculdade de Medicina, Universidade de São Paulo and Hospital e Maternidade Santa Joana Ethics Committee approval, participated in this prospective, randomized and double blind study 69 healthy term pregnant women in active labor without obstetric or fetal complications.

After monitoring with ECG, noninvasive blood pressure and pulse oximetry, patients received volume expansion with 250 $\mathrm{mL}$ lactated Ringer' solution. Combined spinal-epidural anesthesia was induced with patients in the sitting position in $L_{2}-L_{3}$ or $L_{3}-L_{4}$ interspace using the needle-through-needle technique. Anesthetic solution was only administered after passive CSF reflux through needle's gun. Epidural catheter was inserted immediately after. After puncture, patients were placed in the supine position with uterine displacement to the left. Anesthetic solutions were prepared by an anesthesiologist not involved with patients care.

When analgesia was requested, patients were randomly distributed in 3 groups receiving:

- Control Group ( $n=23$ ): spinal $2.5 \mathrm{mg}$ of $0.5 \%$ hyperbaric bupivacaine associated to $1 \mathrm{~mL}$ saline solution;

- Group S2.5 ( $n=23)$ : spinal $2.5 \mu \mathrm{g}$ sufentanil associated to $2.5 \mathrm{mg}$ of $0.5 \%$ hyperbaric bupivacaine and $0.5 \mathrm{~mL}$ saline solution;

- Group S5 ( $n=23)$ : spinal $5 \mu$ g sufentanil associated to 2.5 $\mathrm{mg}$ of $0.5 \%$ hyperbaric bupivacaine.

Pain and possible side effects (nausea, vomiting, pruritus) were evaluated at analgesia induction, at 5-minute intervals in the following 15 minutes and then at 15 -minute intervals until delivery.

Pain was evaluated through a visual analog scale (VAS) from 0 to $10 \mathrm{~cm}(0=$ no pain and $10=$ unbearable pain $)$ and by an anesthesiologist blind to the group the patient belonged to. After first 15 minutes, if pain was higher than $3 \mathrm{~cm}, 4 \mathrm{~mL}$ bolus of increasing $0.125 \%$ and $0.25 \%$ bupivacaine concentrations were administered through epidural catheter at 15-minute intervals until pain was controlled (below $3 \mathrm{~cm}$ ).

The study was ended at first rescue analgesia request or at delivery.

For statistical analysis ANOVA was used to compare age, weight, height, initial dilatation and analgesia duration among 
groups; Kruskal-Wallis test was used for initial VAS and fifteen minutes after spinal injection; Chi-square test was used for delivery before rescue analgesia; and Fisher's Exact test was used to compare nausea, vomiting, pruritus and rescue analgesia in the first 15 minutes, considering significant $p<0.05$.

\section{RESULTS}

Groups were similar in age, weight, height (Table I) and mean initial cervical dilatation (Table II).

Groups receiving $2.5 \mu \mathrm{g}$ or $5 \mu \mathrm{g}$ sufentanil had longer analgesia duration with lower need for rescue analgesia after the first 15 minutes as compared to Control Group (Table II).

Table I - Demographics Data (Mean \pm SD)

\begin{tabular}{lccc}
\hline Groups & $\begin{array}{c}\text { Control } \\
(\mathrm{n}=23)\end{array}$ & $\begin{array}{c}\mathrm{S} 2.5 \mu \mathrm{g} \\
(\mathrm{n}=23)\end{array}$ & $\begin{array}{c}\mathrm{S} 5 \mu \mathrm{g} \\
(\mathrm{n}=23)\end{array}$ \\
\hline Age (years) & $26.7 \pm 6.6$ & $28.4 \pm 7.1$ & $24.5 \pm 6.4$ \\
Weight $(\mathrm{kg})$ & $69.9 \pm 11.3$ & $71.5 \pm 10.3$ & $71.7 \pm 16.0$ \\
Height $(\mathrm{m})$ & $1.60 \pm 0.1$ & $1.59 \pm 0.1$ & $1.60 \pm 0.1$ \\
\hline
\end{tabular}

Table II - Initial Cervical Dilatation, Analgesia Duration and Efficacy (VAS $<3 \mathrm{~cm}$ ) after 15 minutes

\begin{tabular}{lccc}
\hline Groups & $\begin{array}{c}\text { Control } \\
(\mathrm{n}=23)\end{array}$ & $\begin{array}{c}\mathrm{S} 2.5 \mu \mathrm{g} \\
(\mathrm{n}=23)\end{array}$ & $\begin{array}{c}\mathrm{S} 5 \mu \mathrm{g} \\
(\mathrm{n}=23)\end{array}$ \\
\hline Initial dilatation $(\mathrm{cm})$ & $5.7 \pm 1.6$ & $5.8 \pm 1.7$ & $5.4 \pm 1.9$ \\
Analgesia duration $(\mathrm{min})$ & $35.9 \pm 21.6^{*}$ & $67.2 \pm 38.6$ & $78.9 \pm 38.7$ \\
VAS $<3 \mathrm{~cm}$ after 15' $(\%)$ & $69.6^{*}$ & 100 & 95.6 \\
\hline
\end{tabular}

* $p<0.05$, Control Group $<$ Group S2.5 = Group S5

Table III - Incidence of Nausea, Vomiting and Pruritus (Mild or Severe)

\begin{tabular}{lccc}
\hline Groups & $\begin{array}{c}\text { Control } \\
(\mathrm{n}=23)\end{array}$ & $\begin{array}{c}\text { S2.5 } \mu \mathrm{g} \\
(\mathrm{n}=23)\end{array}$ & $\begin{array}{c}\text { S5 } \mu \mathrm{g} \\
(\mathrm{n}=23)\end{array}$ \\
\hline Nausea (\%) & 4 & 4 & 9 \\
Vomiting (\%) & 4 & 4 & 4 \\
Mild pruritus (\%) & $0^{*}$ & 34.8 & 47.8 \\
Severe pruritus (\%) & 0 & 0 & 8.7 \\
\hline
\end{tabular}

* $p<0.05$, Group S2.5 = Group S5 $>$ Control Group

Figure 1 shows the percentage of patients with VAS below 3 $\mathrm{cm}$ as a function of time. There has been a higher number of deliveries before rescue analgesia in Groups S2.5 and S5 as compared to Control Group (Figure 2).

Mean VAS scores at analgesia installation were similar among groups. However it has been observed that 15 minutes after initial spinal anesthetic injection, mean pain scores in Control Group were significantly higher as compared to groups receiving 2.5 and $5 \mu \mathrm{g}$ sufentanil (Figure 3 ).

There were no statistically significant differences in nausea and vomiting among groups. Mild pruritus was higher in groups S2.5 and S5 as compared to Control Group. There has been severe pruritus in two Group S5 patients without statistical difference in this variable among groups (Table III).

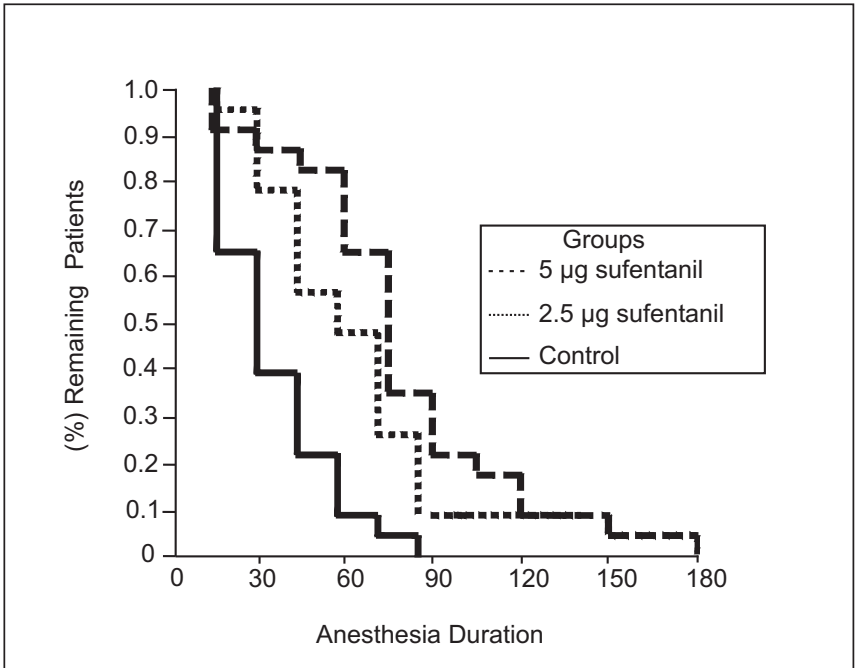

Figure 1 - Percentage of Patients with Effective Analgesia (VAS < 3) as a Function of Time

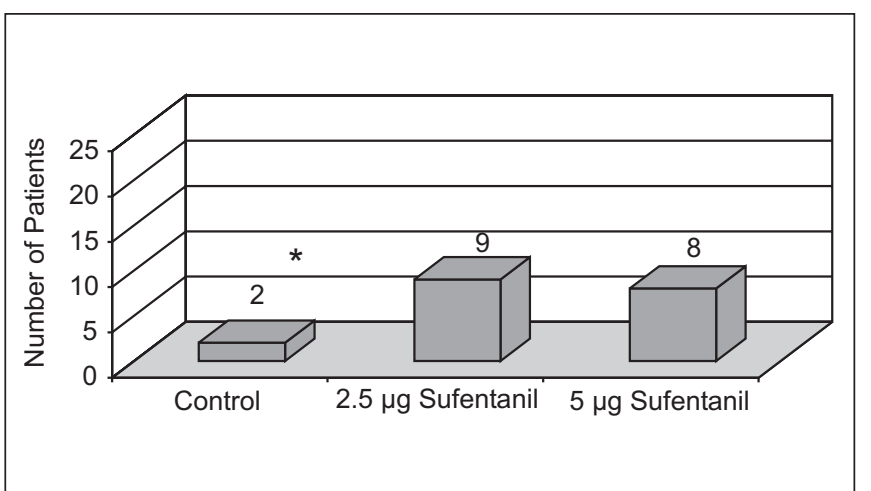

Figure 2 - Number of Deliveries before Analgesic Complementation Need

${ }^{*} \mathrm{p}<0.05$, Control Group $<$ Group S2.5 = Group S5

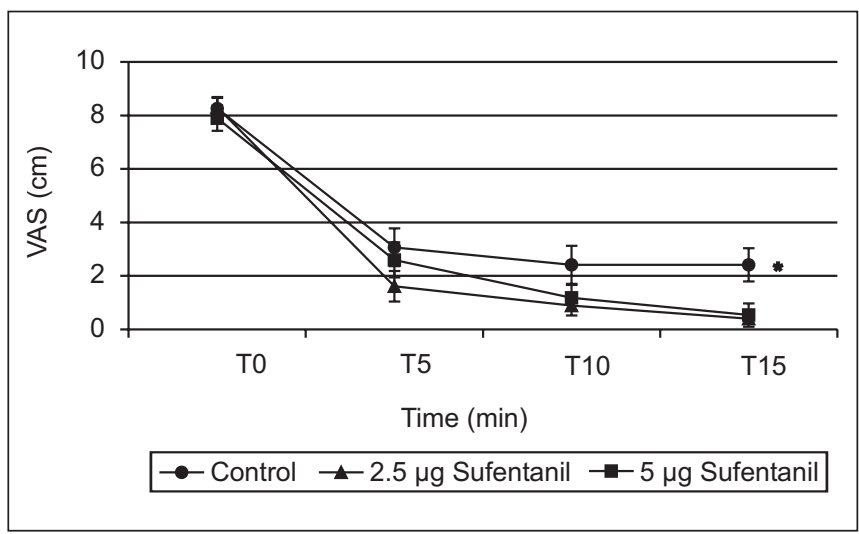

Figure 3 - VAS $(\mathrm{cm})$ before and Fifteen Minutes after Spinal Injection ${ }^{*} p<0.05$, Control Group $>$ Group S2.5 = Group S5

Revista Brasileira de Anestesiologia Vol. 54, № 2, Março - Abril, 2004 


\section{DISCUSSION}

Our results have shown that the association of $2.5 \mu \mathrm{g}$ or $5 \mu \mathrm{g}$ sufentanil to spinal hyperbaric bupivacaine for labor analgesia provides longer duration and better analgesia as compared to local anesthetic alone. These data are in line with Wong et al. ${ }^{4}$ who have studied dose-response of opioid/local anesthetics combination and have also concluded that the association of different sufentanil doses $(0.25 ; 5 ; 7.5$ and 10 $\mu \mathrm{g})$ to isobaric bupivacaine would improve quality and prolong labor analgesia duration.

The major difference between both studies is the baricity of spinal local anesthetics. The association of 0.5 or $1 \mathrm{~mL}$ sufentanil (density $=0.9933 \mathrm{~g} \cdot \mathrm{mL}^{-1}$ ) to $0.5 \mathrm{~mL}$ of $0.5 \%$ hyperbaric bupivacaine (density $=1.026 \mathrm{~g} \cdot \mathrm{mL}^{-1}$ ) at $20^{\circ} \mathrm{C}$ produces hyperbaric solutions (density $=1.002935 \mathrm{~g} \cdot \mathrm{mL}^{-1}$ ). Wong et al. ${ }^{4}$ in associating different sufentanil doses to hypobaric bupivacaine, have worked with isobaric solutions. It is known that changes in baricity of spinal anesthetic solutions, especially sufentanil, may significantly change anesthetic solution spread dynamics in the spinal space and, as a consequence, its clinical effects ${ }^{5}$.

Ferouz et al. ${ }^{6}$ have shown that $1 \mathrm{~mL}$ of $10 \%$ dextrose associated to $1 \mathrm{~mL}$ sufentanil $\left(10 \mu \mathrm{g} \cdot \mathrm{mL}^{-1}\right)$ thus producing hyperbaric sufentanil solution, would determine less side effects (especially severe pruritus) but would impair analgesic quality. So, they have suggested that side effects would depend on the supraspinal action of opioids and that the analgesic action would depend both on the spinal and supraspinal action.

Although not directly comparing analgesic efficacy of hypobaric and hyperbaric sufentanil-containing solutions to bupivacaine, it is possible that potential analgesia quality impairment caused by hyperbaric sufentanil has been corrected by the association to local anesthetics, since both drugs would have additive and synergistic effects on pain. It is our routine to administer opioids associated to local anesthetics, since in our practice labor analgesia is often induced at the end of the first labor stage and beginning of the second. Previous studies have shown that spinal opioids alone are not effective in relieving labor pain, especially as the patient gets closer to the second labor stage ${ }^{7-10}$.

However it is worth mentioning that in this study analgesia was always based on patients' request and not on cervical dilatation. Although mean cervical dilatation at analgesia request was approximately $5 \mathrm{~cm}$, there has been wide individual variation (1 to $9 \mathrm{~cm}$ ), which reflects individual differences in pain tolerance during labor.

We decided not to perform the test dose with $3 \mathrm{~mL}$ of $1.5 \%$ lidocaine and 1:200,000 epinephrine immediately after spinal anesthetic injection and further epidural catheter insertion as proposed by Wong et al. ${ }^{4}$ because test dose could contribute to analgesia.

Test dose could improve the quality of analgesia with low spinal sufentanil and bupivacaine doses by the direct action of lidocaine in the epidural space, or indirectly, by interfering with spread dynamics of recently injected anesthetics and not yet fixed within the spinal space (mass effect and transmission of epidural pressure to the spinal space).

Cardoso et al. ${ }^{11}$, evaluating epidural pressure changes promoted by local anesthetics injected in different volumes and rates, have shown that even low volumes such as those used for test dose, when rapidly injected, determine higher epidural space peaks than the administration of higher volumes at lower rates.

It should be also mentioned that instantaneous checking of correct epidural catheter position does not assure its permanence in the same place during future manipulations. There are in the literature several cases of intravascular and spinal migration of epidural catheters previously well positioned. In addition, in labor analgesia, complications of intravascular local anesthetic injections by epidural catheter malposition are uncommon, because the anesthesiologist is working with extremely low local anesthetic doses and concentrations.

As to side effects, it has been observed that when sufentanil dose was decreased from 5 to $2.5 \mu \mathrm{g}$, there has been no change in nausea, vomiting, mild pruritus, severe pruritus and analgesia duration. These data are in line with Wong et al. ${ }^{4}$ who have also found no differences in the incidence of nausea, vomiting, pruritus and analgesia duration after the administration of different spinal sufentanil doses $(2.5 ; 5 ; 7.5$ and $10 \mu \mathrm{g})$. In both studies, differences in the incidence of side effects and analgesia duration were only found between groups receiving sufentanil associated to bupivacaine as compared to control groups where labor analgesia was induced with local anesthetics alone.

It is however important to stress that sample size was not enough to show possible differences between both sufentanil doses. Power analysis for side effects-related variables has shown that in order to detect approximately $13 \%$ difference in pruritus, two groups of 198 patients each would have to be studied.

It has also been observed that the number of deliveries before the need for epidural complementation was higher in groups receiving sufentanil as compared to control. This fact does not necessarily indicate faster cervical dilatation with sufentanil, but is possibly related to longer analgesia duration in those groups as compared to control group.

Although respiratory depression has already been reported with spinal sufentanil ${ }^{12-14}$, we have not observed respiratory depression after spinal sufentanil and although the small sample size to draw any conclusion, it is possible that factors contributing for the absence of such complication were: non utilization of any other systemic drug depressing central nervous system; anesthetic solutions were prepared as from sufentanil's parent solution for spinal use, which contains just $5 \mu \mathrm{g}$ of the drug per $\mathrm{mL}$ and not as from the solution for intravenous use, which contains $50 \mu \mathrm{g}$ of the drug per $\mathrm{mL}$ (thus preventing potential dilution errors); sufentanil doses used in our study were lower $(2.5$ and $5 \mu \mathrm{g})$ than those used when respiratory depression has been observed $(10 \mu \mathrm{g})$; and the use of hyperbaric solutions which probably limit cephalad spread of drugs to the spinal space ${ }^{6}$. 
In conclusion, the association of different sufentanil doses to spinal hyperbaric bupivacaine improves labor analgesia quality and duration. When associated to $2.5 \mathrm{mg}$ hyperbaric bupivacaine there is no advantage in administering sufentanil doses higher than $2.5 \mu \mathrm{g}$.

\section{REFERÊNCIAS - REFERENCES}

01. Herman NL, Calicott R, Van Decar TK et al - Determination of the dose-response relationship for intrathecal sufentanil in laboring patients. Anesth Analg, 1997;84:1256-1261.

02. Arkoosh VA, Cooper M, Norris MC et al - Intrathecal sufentanil dose response in nulliparous patients. Anesthesiology, 1998; 89:364-370

03. Campbell DC, Camann WR, Datta S - The addition of bupivacaine to intrathecal sufentanil for labor analgesia. Anesth Analg, 1995;81:305-309.

04. Wong CA, Scavone BM, Loffredi M et al - The dose-response of intrathecal sufentanil added to bupivacaine for labor analgesia. Anesthesiology, 2000;92:1553-1558.

05. Nicol ME, Holdcroft A - Density of intrathecal agents. $\mathrm{Br} \mathrm{J}$ Anaesth, 1992;68:60-63.

06. Ferouz F, Norris MC, Arkoosh VA et al - Baricity, needle direction, and intrathecal sufentanil labor analgesia. Anesthesiology, 1997;86:592-598.

07. Camann WR, Denney RA, Holby ED et al - A comparison of intrathecal, epidural, and intravenous sufentanil for labor analgesia. Anesthesiology, 1992;77:884-887.

08. Camann WR, Minzter BH, Denney RA et al - Intrathecal sufentanil for labor analgesia. Effects of added epinephrine. Anesthesiology, 1993;78:870-874.

09. Honet JE, Arkoosh VA, Norris MC et al - Comparison among intrathecal fentanyl, meperidine, and sufentanil for labor analgesia. Anesth Analg, 1992;75:734-739.

10. Norris MC, Fogel ST, Holtmann B et al - Intrathecal sufentanil (5 vs. $10 \mu \mathrm{g}$ ) for labor analgesia: efficacy and side effects. Reg Anesth Pain Med, 1998;23:252-257.

11. Cardoso MMSC, Carvalho JCA - Epidural pressures and spread of $2 \%$ lidocaine in the epidural space: influence of volume and speed of injection of the local anesthetic solution. Reg Anesth, 1998;23:14-19.

12. Fournier R, Gamulin Z, Van Gessel E - Respiratory depression after $5 \mu \mathrm{g}$ of intrathecal sufentanil. Anesth Analg, 1998;87: 1377-1378.
13. Hays RL, Palmer CM - Respiratory depression after intrathecal sufentanil during labor. Anesthesiology, 1994;81:511-512.

14. Carvalho JCA - Respiratory arrest following intrathecal sufentanil. Can J Anesth, 1999;46:198-199.

\section{RESUMEN}

Yamaguchi ET, Carvalho JCA, Fonseca US, Hirahara JT, Cardoso MMSC - Sufentanil Subaracnóideo Asociado a la Bupivacaína Hiperbárica para Analgesia de Parto: Es Posible Reducir la Dosis del Opioide?

JUSTIFICATIVA Y OBJETIVOS: La adición de bupivacaína isobárica a dosis menores de sufentanil por vía subaracnóidea promueve analgesia de cualidad satisfactoria, con menor incidencia de efectos colaterales. El objetivo del estudio fue evaluar la calidad de la analgesia y la incidencia de efectos colaterales de dosis reducidas de sufentanil subaracnóideo asociados a bupivacaína hiperbárica en analgesia de parto.

MÉTODO: Fueron estudiadas prospectivamente 69 embarazadas de término en trabajo de parto. Las embarazadas fueron aleatoriamente divididas en tres grupos que recibieron, en el espacio subaracnóideo, la combinación de $2,5 \mathrm{mg}$ de bupivacaína hiperbárica y $1 \mathrm{~mL}$ de solución fisiológica (Grupo Control); $2,5 \mathrm{mg}$ de bupivacaína hiperbárica y 2,5 $\mu \mathrm{g}$ de sufentanil (Grupo S2,5) o 2,5 mg de bupivacaína hiperbárica y 5 $\mu g$ de sufentanil (Grupo S5). El dolor, de acuerdo con la escala analógico visual (EAV) de dolor y la incidencia de efectos colaterales fueron evaluadas a cada 5 minutos en los primeros quince minutos y a seguir a cada 15 minutos hasta el nacimiento. El estudio terminaba con el nacimiento, o cuando la paciente solicitaba medicación analgésica de rescate (EAV> $3 \mathrm{~cm}$ ).

RESULTADOS: Los grupos S2,5 y S5 presentaron mayor duración de analgesia (67,2 \pm 38,6 y 78,9 \pm 38,7 minutos, respectivamente) y mayor porcentaje de pacientes con analgesia efectiva $(100 \%$ y $95,6 \%$, respectivamente) que el grupo Control, en el cual la duración media de analgesia fue de 35, $9 \pm$ 21,6 minutos $(p<0,05)$ y el porcentaje de pacientes con analgesia efectiva fue de $69,6 \%(p<0,05)$.

CONCLUSIONES: La adición de sufentanil a la bupivacaína hiperbárica mejora la calidad y prolonga la duración de la analgesia. Cuando asociado a $2,5 \mathrm{mg}$ de bupivacaína hiperbárica, no hay ventaja de administrar dosis superior a 2,5 $\mu \mathrm{g}$ de sufentanil para alivio del dolor del trabajo de parto. 